\title{
Expanding TAVI to Low and Intermediate Risk Patients
}

\author{
Lisa Voigtländer ${ }^{1}$ and Moritz Seiffert ${ }^{1,2 *}$ \\ ${ }^{1}$ Department of General and Interventional Cardiology, University Heart Center Hamburg, Hamburg, Germany, ${ }^{2}$ Partner site \\ Hamburg/Kiel/Lübeck, DZHK, German Centre for Cardiovascular Research, Hamburg, Germany
}

TAVI has become the standard treatment in patients at increased surgical risk and is increasingly being performed in patients at intermediate to low surgical risk. While non-inferiority has been demonstrated in intermediate risk patients, several challenges - particularly with regard to valve durability - need to be addressed before expansion to lower risk and younger patients can be recommended on a broad basis. Current trends, trials results, and remaining challenges are summarized and discussed in the light of updated treatment guidelines.

Keywords: TAVI, TAVR, intermediate risk, low risk, aortic valve stenosis

\section{INTRODUCTION}

OPEN ACCESS

Edited by:

Crochan John O'Sullivan,

Triemli Hospital, Switzerland

Reviewed by:

Masahiko Asami,

Inselspital, Bern University Hospital,

Switzerland

Luis Nombela-Franco,

Hospital Clínico San Carlos, Spain

*Correspondence:

Moritz Seiffert

m.seiffert@uke.de

Specialty section:

This article was submitted to Structural Interventional Cardiology, a section of the journal

Frontiers in Cardiovascular Medicine

Received: 02 April 2018

Accepted: 25 June 2018

Published: 12 July 2018

Citation:

Voigtländer L and Seiffert M (2018)

Expanding TAVI to Low and

Intermediate Risk Patients.

Front. Cardiovasc. Med. 5:92.

doi: 10.3389/fcvm.2018.00092
Severe aortic valve stenosis (AS) represents the most common valvular heart disease in developed countries. Since its prevalence is associated with increasing age, a growing disease burden is expected in the future considering an aging patient population (1). Surgical aortic valve replacement (SAVR) - the traditional standard of care for patients with severe symptomatic AS-is increasingly complemented by transcatheter aortic valve implantation (TAVI). After the first TAVI procedure in 2002 (4), the number of procedures has increased exponentially in the past years and has recently outperformed the number of isolated SAVR per year in Germany (5). Several prospective randomized trials demonstrated non-inferiority for TAVI compared to SAVR in patients at high surgical risk $(6,7)$. More recently, three additional trials reported non-inferiority of TAVI in intermediate-risk patients (Figure 1, Table 1) (8-10). Current debates focus on the expansion of TAVI as the standard of care for the treatment of patients with AS and low to intermediate operative risk.

\section{ASSESSMENT OF OPERATIVE RISK}

What are criteria and cutoffs for low to intermediate operative risk? Objective risk estimation remains the Achilles' heel for the evaluation of individual treatment options and overall comparison of clinical trial results. A multitude of relevant clinical and anatomical factors effectively influence operative complexity, complicating precise risk calculation in these patients. All of the widely-used risk stratification tools (STS-PROM, logistic EuroSCORE, EuroSCORE II) entail significant limitations in predicting operative mortality $(11,12)$. In the absence of a perfectly reliable risk model, the STS-PROM has mostly been applied for individual risk assessment and for comparison of trials results. In the past, operative risk was classified as high (STS-PROM >8\%), intermediate (STS-PROM of 4-8\%), and low (STS-PROM <4\%).

Abbreviations: AS, Aortic stenosis; LogES, Logistic EuroSCORE; PVL, Paravalvular leakage; SAVR, Surgical aortic valve replacement; STS-PROM, Society of Thoracic Surgeons' Predicted Risk of Operative Mortality; TAVI, Transcatheter aortic valve implantation; THV, Transcatheter heart valve. 


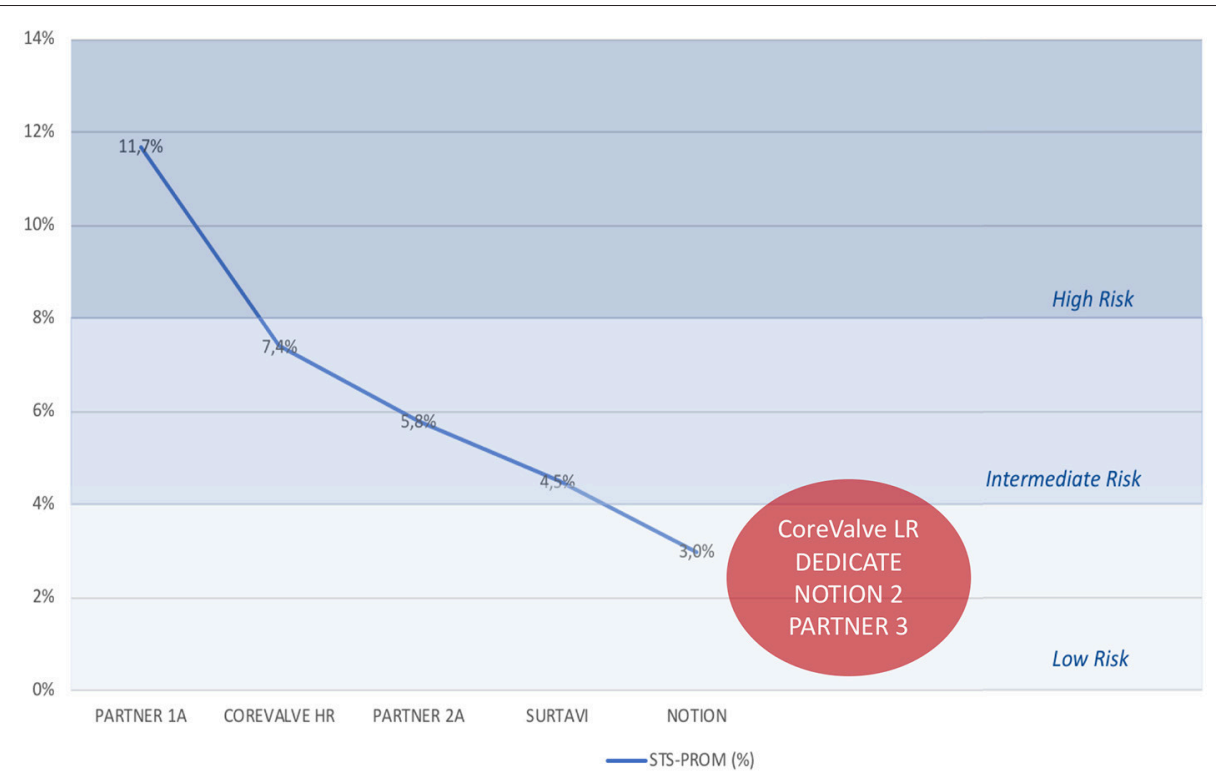

FIGURE 1 | Evolution of operative risk in major trials. Decline in operative risk, as assessed by the STS-PROM score, in major randomized trials comparing TAVI and SAVR (6-10) and anticipated low to intermediate risk of currently active trials (CoreValve LR, DEDICATE, NOTION 2, PARTNER 3). SAVR, surgical aortic valve replacement; STS-PROM, Society of Thoracic Surgeons' Predicted Risk of Operative Mortality; TAVI, transcatheter aortic valve implantation.

However, important additional factors, e.g. active malignancy, frailty, porcelain aorta, chest wall radiation, liver cirrhosis, or neurological impairment, were not comprehensively integrated in these risk models. In addition, treatment decisions may differ in elderly patients without comorbidities (low operative risk despite advanced age) and young patients with significant comorbidities (increased operative risk despite young age). The 2017 ESC/EACTS guidelines for the management of valvular heart disease incorporate these difficulties and opt for a more differentiated approach to operative risk and choice of treatment modality (13).

\section{TREATMENT SELECTION ACCORDING TO CURRENT GUIDELINES}

To help navigate the choice of treatment modality in patients with low to intermediate surgical risk, European (13) and American (14) guidelines were recently updated. In general, the indication for TAVI was expanded to intermediate risk patients in both versions on the basis of three major trials (810). American guidelines in its current version consider TAVI a reasonable alternative to SAVR in patients at intermediate operative risk (STS-PROM $\geq 4 \%$ ), depending on patientspecific procedural risks, values, and preferences (14). European guidelines emphasize, that the treatment selection (TAVI or SAVR) in patients at increased surgical risk (STS-PROM $\geq 4 \%$, logistic EuroSCORE $\geq 10 \%$ or risk factors not considered in these algorithms) should be made by the Heart Team on an individualized basis (13). According to the guideline's authors, factors in favor of a catheter-based approach include patient age $\geq 75$ years, prior cardiac surgery, frailty, restricted mobility or anticipated prolonged rehabilitation, favorable transfemoral access, prior chest radiation, porcelain aorta, severe chest deformation, or expected prosthesis-patient mismatch. Other aspects, e.g. patient age $<75$ years, suspicion of endocarditis, unfavorable anatomy for TAVI (access, low coronary take-off, unfavorable aortic root, valvular, or annular anatomy), and concomitant cardiac conditions that require additional surgical treatment favor SAVR. Overall, SAVR remains the standard therapy for patients $<75$ years of age with low surgical risk at current as long-term durability data for THV remain insufficient. In the absence of a perfect risk assessment, both guidelines emphasize the integral role of the interdisciplinary heart team in patient evaluation, assessment of technical suitability, and identification of the appropriate treatment modality $(13,14)$.

\section{EVIDENCE FROM INTERMEDIATE-RISK TRIALS OR REGISTRIES}

Essential evidence for the expansion of TAVI for the treatment of intermediate risk patients stems from three prospective randomized trials and reports from major contemporary registries.

\section{Registries}

Several large-scale nationwide registries evaluated outcomes and trends in the treatment of aortic valve stenosis. Long before first results from prospective randomized intermediate-risk trials were available, large registries had already reported a paradigm shift of TAVI towards lower risk patients: According to the 


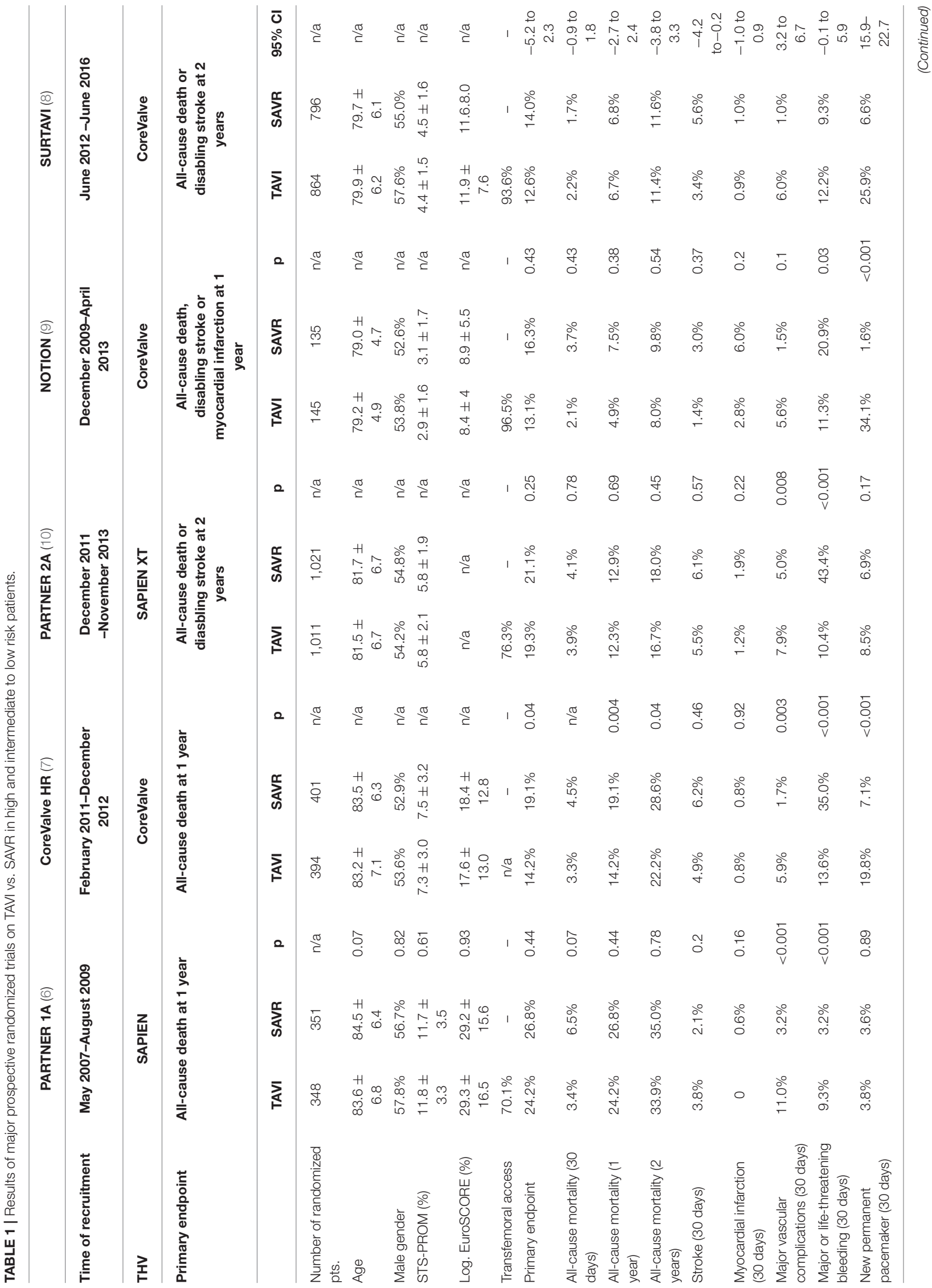




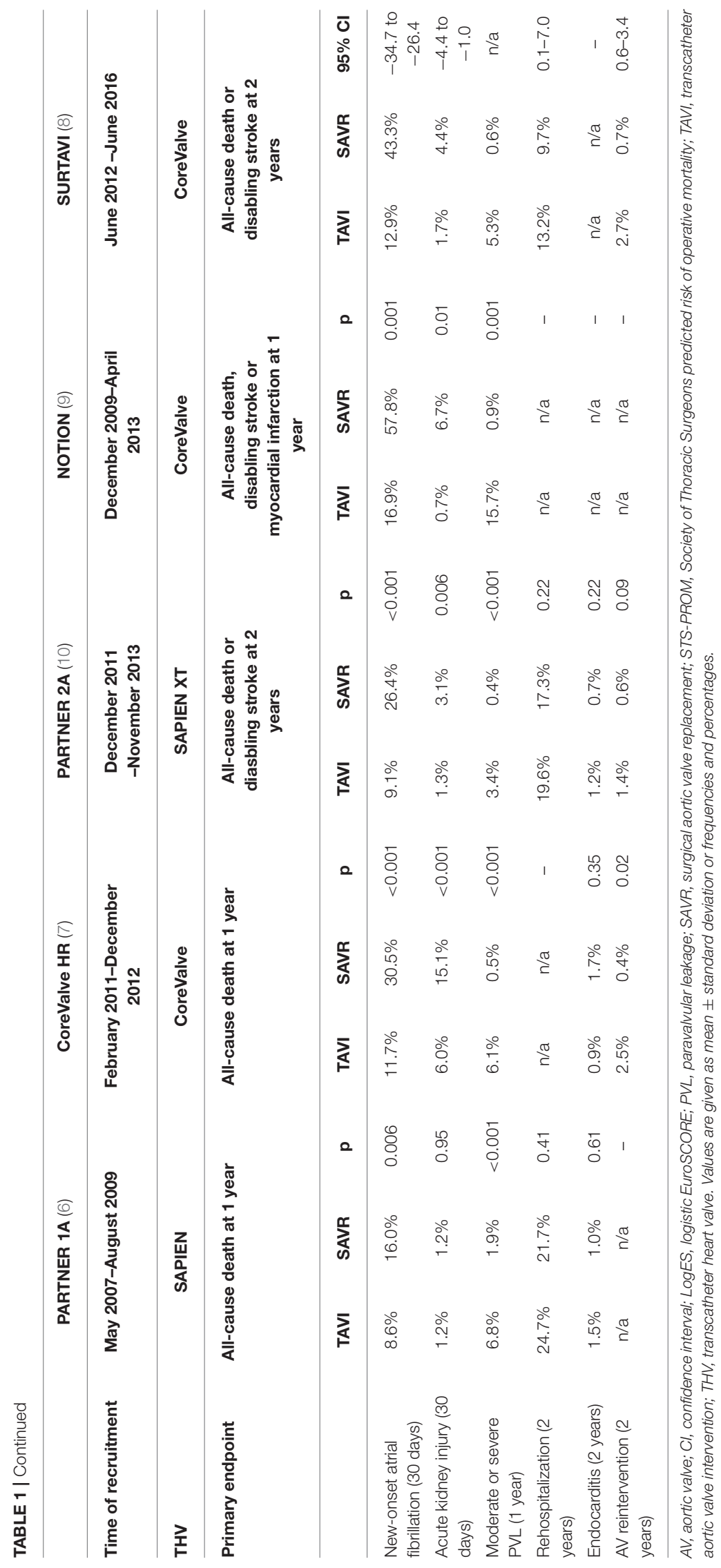


compulsory German quality assurance registry on aortic valve replacement (AQUA), the number of annual TAVI procedures in Germany increased 20-fold from 2008 to 2014 while the number of SAVR procedures slowly declined (15). Interestingly, operative risk, as assessed by the logistic EuroSCORE, decreased significantly over the years with a larger percentage of patients at low to intermediate risk in the later years $(\log \mathrm{ES}<10 \%: 18.9 \%$ [2012] vs. $25.9 \%$ [2014]). This was followed by a drop in hospital mortality after TAVI during the observation period (2008: 10.4\%, 2014: 4.2\%) (15).

Similar trends were observed in the German Aortic Valve Registry (GARY), which included a total of 15,964 patients undergoing TAVI between 2011 and 2013 (16). Over the years, a significant regression in risk profiles (logES 20.2\% [2011] to $16.9 \%$ [2013]; STS-PROM: 5.2\% [2011] to 4.9 [2013], both $p<0.001)$, periprocedural complications and in-hospital mortality (5.9\% [2011] to $4.9 \%$ [2013], $p=0.078)$ were observed (16).

The Society of Thoracic Surgeons (STS)/American College of Cardiology Transcatheter Valve Therapy (TVT) Registry collected data from 54,782 TAVI procedures performed in the United States from 2012 to 2015. The volume of annual TAVI procedures increased from 4,627 to 24,808 in this time window (17). While the median STS-PROM decreased from 7.1 to $6.3 \%$ (2012 vs. $2015, p<0.001$ ), a subsequent decline of 30-day mortality (7.5\% [2012] vs. $4.6 \%$ [2015], $p<0.0001)$, stroke (2.3\% [2012] vs. $1.9 \%$ [2015], $p=0.0264)$, or moderate/severe PVL (2012:10.8\% [2012] vs. $6.2 \%$ [2015], $p<0.0001)$ was observed (17).

A shift in patients' disease severity and advancements in procedural and technical aspects over the past years have most likely contributed to these consistent improvements of outcomes after TAVI. However, a comparison of treatment modalities from these registries' results is impeded by very different risk profiles in the treatment groups, calling for appropriate randomized trials.

\section{Randomized Trials}

In addition to several real-world registries, few comprehensivebut highly selective-industry-sponsored trials evaluated outcomes after TAVI in different risk categories (see Table $\mathbf{1}$ for selected results, Figure for risk profile). Results of intermediate risk trials are discussed in the following.

The first randomized trial to evaluate TAVI in low to intermediate risk patients was the Nordic STACCATO trial. It started patient recruitment as early as 2008 and aimed to compare transapical TAVI to SAVR in operable patients $\geq 75$ years of age (18). Due to an excess of serious adverse events in the transapical TAVI arm, the study was prematurely terminated after inclusion of 70 patients. The trial was heavily criticized for its design, including only a transapical TAVI arm.

One year later, the NOTION (Nordic Aortic Valve Intervention) trial (9) started recruitment. NOTION randomized 280 patients $\geq 70$ years of age with severe aortic stenosis to TAVI with the Medtronic CoreValve THV or SAVR at three Nordic centers (TAVI:145 patients; SAVR: 135 patients). Mean STSPROM was $2.9 \pm 1.6 \%$ in TAVI and $3.1 \pm 1.7 \%$ in SAVR patients. The access route was transfemoral in $96.5 \%$ of TAVI cases. The composite primary endpoint (all-cause mortality, stroke or myocardial infarction) and all-cause mortality were similar in both groups (13.1\% [TAVI] vs. $16.3 \%$ [SAVR] and $4.9 \%$ [TAVI] vs. $7.5 \%$ [SAVR], $p=0.38$ ). Periprocedural complications differed according to treatment arm with an access of major/lifethreatening bleeding (11.3\% [TAVI]) vs. 20.9 [SAVR]), acute kidney injury stage $3(0.7 \%$ [TAVI] vs. $6.7 \%$ [SAVR]), and new-onset or worsening atrial fibrillation (16.9 [TAVI] vs. $57.8 \%$ [SAVR], $p<0.001)$ in the SAVR arm. Rates of permanent pacemaker implantation (34.1\% [TAVI] vs. $1.6 \%$ [SAVR], $p<0.001$ ) and PVL (moderate/severe at 1 year: 15.7\% [TAVI] vs. $0.9 \%$ [SAVR]) were observed more frequently in patients treated with TAVI. At the same time, transvalvular gradients and effective orifice areas were in favor of TAVI treatment. Recent 5 -year data confirmed non-inferiority of TAVI compared to SAVR regarding the composite endpoint (TAVI: 39.2\%; SAVR $35.8 \% ; p=0.78)(2)$ and the 5 -year all-cause mortality of $27.7 \%$ was the lowest 5 -year mortality rate ever reported in a TAVI population. NOTION was the first prospective randomized trial to generate data on TAVI in intermediate to low risk patients. However, the small sample size and the large rate of screening failures challenge the "all-comers" character of the trial.

At a larger scale, the PARTNER 2A trial randomized 2,032 patients with intermediate surgical risk (STS-PROM score 4$8 \%$ and heart team consensus) to either TAVI with the balloonexpandable SAPIEN XT or SAVR (10). The mean STS-PROM was $5.8 \%$ and almost twice as high as in the NOTION trial. The composite endpoint at 2 years (all-cause death or disabling stroke) was non-inferior in patients treated with TAVI compared to SAVR (TAVI: $19.3 \%$, SAVR: $21.1 \%, p=0.25$ ). A subsequent subgroup analysis even demonstrated superiority for the transfemoral cohort compared to SAVR (16.3 vs. $20 \%$, $p=0.04)$. At 2 years of follow-up, a higher incidence of lifethreatening/disabling bleeding ( 47.0 vs. $17.3 \%, p<0.001$ ), acute kidney injury stage $3(6.2$ vs. $3.8 \%, p=0.02)$, and new onset atrial fibrillation ( 27.3 vs. $11.3 \%, p<0.001)$ were reported after SAVR while patients after TAVI had a higher risk for major vascular complications ( 8.6 vs. $5.5 \%, p=0.006$ ). Interestingly, rates of permanent pacemaker implantations were not significantly different in both groups in this trial. An overall faster recovery and shorter hospitalization (in-hospital: median 6 vs. 9 days, ICU: median 2 vs. 4 days, $p<0.001$ for both) were observed after TAVI. While lower transprosthetic gradients were reported in the TAVI arm, the rate of moderate/severe PVL was significantly higher compared to SAVR ( 8.0 vs. $0.6 \%, p<0.001$ ) and a trend towards more aortic valve re-interventions was observed after TAVI at 2 years ( 1.4 vs. $0.6 \%, p=0.09$ ). This observation has to be followed closely as the TAVI indication is expanded to younger patients. Of note, $14.5 \%$ of patients in the SAVR arm underwent concomitant coronary artery bypass graft surgery for significant coronary artery disease.

After a recruitment period of almost 4 years, the SURTAVI trial (8) recently reported results of 1,764 patients at intermediate surgical risk (predicted 30-day operative mortality $3-15 \%$ ). The mean STS-Score was $4.5 \pm 1.6 \%$ and thus in between the PARTNER 2A and NOTION trials. Patients were randomized 1:1 to TAVI with the self-expanding CoreValve or CoreValve Evolut 
$\mathrm{R}$ prostheses and SAVR. The primary endpoint, a composite of all-cause death and disabling stroke at 2 years, was similar in both treatment arms (12.6\% [TAVI] vs. 14\% [SAVR], 95\%CI -5.2 to $2.3 \%)$. Again, higher rates of acute kidney injury (4.4 vs. $1.7 \%$ ), new onset atrial fibrillation (43.4 vs. $12.9 \%$ ), and transfusion requirements ( 41.1 vs. $12.5 \%)$ were observed after SAVR. While hemodynamic measures were in favor of TAVI (transprosthetic gradients, effective orifice area), the incidence of PVL (moderate/severe at 1 year: 5.3 vs. $0.6 \%$ ) and the need for pacemaker implantation (25.9 vs. 6.6\%) were lower after SAVR. Quality of life at 2 years was similar in both groups. Aortic valve reintervention was reported more often after TAVI (2.7 vs. $0.7 \%$ at 2 years), although no structural valve deterioration was found in either group.

\section{Currently Active Intermediate to Low Risk Trials}

Building on the results of intermediate-risk trials and registries named above, several prospective randomized trials are currently active, either recruiting patients or in follow-up, to evaluate outcomes after TAVI in patients at low to intermediate operative risk. The results of these trials will determine future guideline recommendations on the treatment of aortic stenosis in low to intermediate risk patients (see Table 2 for major characteristics of these trials).

The PARTNER 3 trial (clinicaltrials.gov NCT02675114) randomly assigns 1,328 patients with low surgical risk (STSPROM $<4 \%$ ) to TAVI with the Sapien 3 device or SAVR. Patients will be followed for 10 years and the primary endpoint is a composite of all-cause mortality, stroke and rehospitalization at 1 year. Results of the primary endpoint are expected to be presented in 2019.

The Medtronic TAVR low risk trial (clinicaltrials.gov NCT02701283) includes 1,200 patients with an STS-PROM $<3 \%$. Patients are randomized to TAVI with the CoreValve or CoreValve Evolut R self-expandable THV or SAVR. Patients will be followed for 10 years and the primary endpoint is a composite of all-cause mortality or disabling stroke at 2 years.

While both studies are industry-sponsored and limited to one THV, two additional investigator-initiated trials have been initiated:

The Nordic NOTION-2 trial (clinicaltrials.gov NCT02825134) aims to randomize 992 low risk patients (STS $<4 \%, \leq 75$ years) to TAVI with any CE-marked device or SAVR. Due to the exclusion of elderly patients, this trial will particularly gain important insights into outcomes of TAVI in younger patients at low risk. Interestingly, combined procedures (SAVR and concomitant CABG or TAVI and PCI) are also included in the trial. The primary endpoint is a composite of all-cause mortality, stroke or myocardial infarction at 1 year. The trial is investigator-initiated but industry-funded.

The DEDICATE trial (DEDICATE-DZHK6, clinicaltrials.gov NCT03112980) is multicenter investigator-initiated and industry-independent study. It is funded by the DZHK (German Center for Cardiovascular Research), the Deutsche Herzstiftung e.V., and supported by German health insurance providers. Overall 1,600 patients at low to intermediate surgical risk (STS-PROM 2-6\%) will be included. As opposed to previous trial designs, DEDICATE aims to investigate a true all-comers patient population and evaluate real-world outcomes. After 1:1 randomization to either TAVI or SAVR, the remaining treatment decisions (e.g. access route, THV type, periprocedural treatment, etc.) are left to the interdisciplinary heart team. All CE-marked devices can be utilized to avoid any potential device-based bias. To account for the increasing importance of long-term data in low risk patients, the primary endpoint was chosen as overall survival after 5 years. Low to intermediate risk patients undergoing aortic valve treatment at the study sites who are not included in the randomized trial will be captured in a nested registry to evaluate an all-comers population.

All of these active trials will add significantly to the current evidence for TAVI in intermediate to low risk patients and allow first insights into long-term results on a broad basis.

\section{REMAINING CHALLENGES}

Within the last decade, TAVI has become the standard of care for high-risk patients with severe and symptomatic AS. It has increasingly been performed in intermediate and also low-risk patients more recently. Particularly for younger and low-risk patients, additional challenges need to be addressed:

\section{Valve Durability and Function}

The unresolved issue of long-term valve durability is probably the key challenge in expanding TAVI to lower risk and younger age patients. Longitudinal echocardiographic evaluation of the PARTNER trials (PARTNER 1A, 1B, and continued access) demonstrated stable hemodynamic results after TAVI over 5 years of follow-up (19). Similar results were reported in other series and for self-expanding transcatheter heart valves (20). Recently results from the Nordic NOTION trial confirmed not only robust hemodynamic data over 5 years of follow-up but also favorable hemodynamics after TAVI compared to SAVR (2). Particularly in patients with smaller aortic annuli, TAVI may yield a lower incidence of patient-prosthesis mismatch, compared to SAVR. However, increased rates of PVL were consistently observed after TAVI compared to SAVR. Due to an adverse effect of significant paravalvular leakage on survival (10), reduction of residual regurgitation will be essential to improve long-term outcomes. Although progress has been made to reduce residual AR after TAVI in recent studies with next-generation devices (21), further improvements will be required to match data from SAVR cohorts.

Additionally, subclinical leaflet thrombosis, its effects on hemodynamic and clinical results need to be evaluated due to a significantly higher incidence after TAVI compared to SAVR (22). Overall, the incidence of structural valve degeneration and aortic valve re-intervention were low but will naturally become an issue as follow-up length and patient numbers increase. Recently published definitions of prosthesis degeneration may aid comprehensive analysis of this important topic $(23,24)$. To eliminate durability concerns after TAVI, very solid durability data available for surgical bioprostheses over the course of more than a decade will need to be matched (25). 
TABLE 2 | Overview of currently active randomized trials on TAVI vs. SAVR in low to intermediate risk patients with severe aortic stenosis.

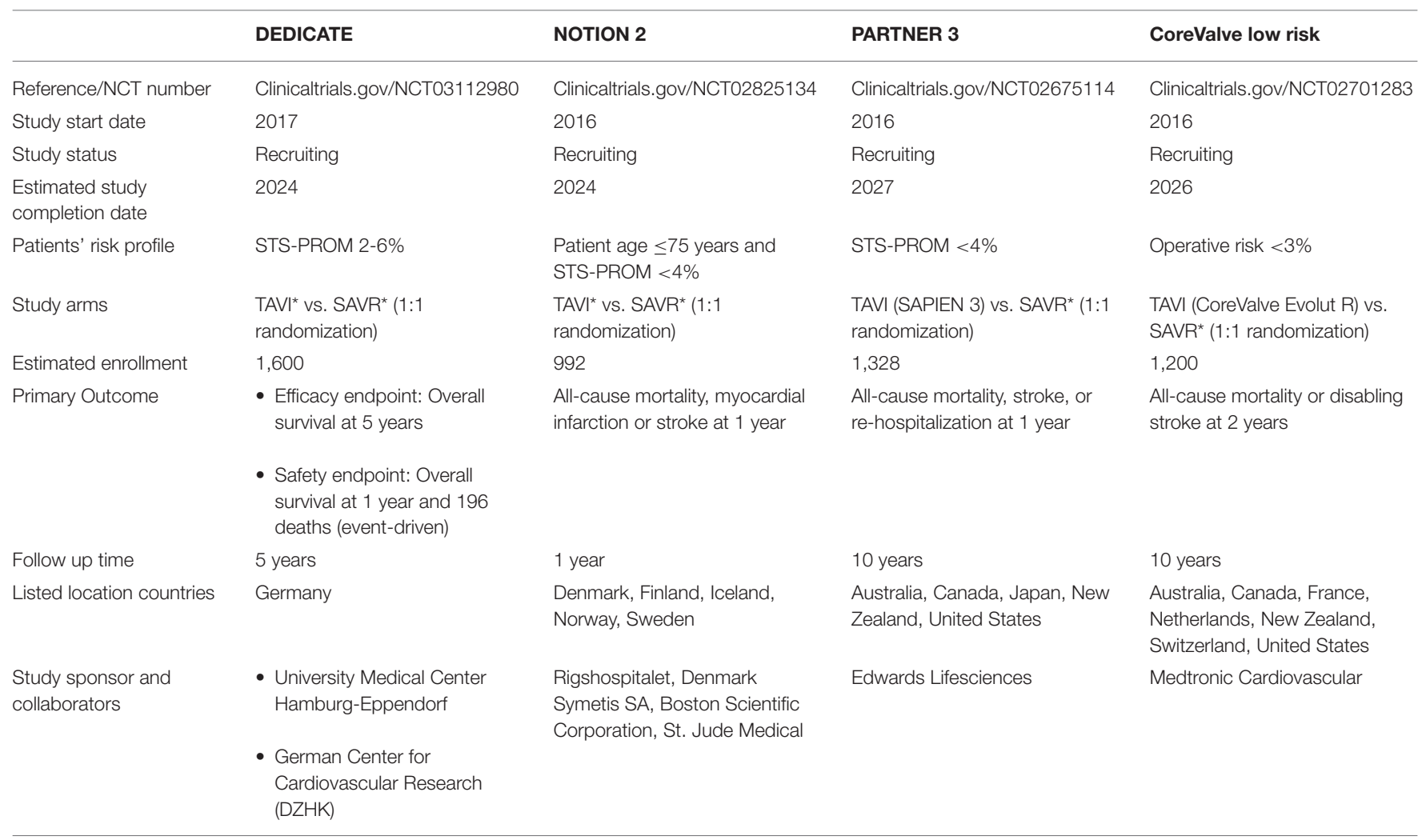

SAVR, surgical aortic valve replacement; STS-PROM, Society of Thoracic Surgeons predicted risk of operative mortality; TAVI, transcatheter aortic valve intervention; "Any commercially available or CE marked device. Information up-to-date as available on clinicaltrials. gov on June 10th, 2018.

Nevertheless, degeneration of THV will occur at some point in patient life, leading to either surgical valve replacement or valve-in-valve procedures. Valve-in-valve procedures have demonstrated encouraging results in patients with degenerated surgical aortic bioprostheses (26). Whether these results can be systematically achieved for valve-in-valve procedures in degenerated THV needs to be demonstrated. Different design features of THV may yield variable results after valve-in-valve implantation, for example with regard to coronary access in degenerated supra-annular THV.

While moving towards younger patients, the prevalence of biscuspid aortic valve disease will inevitably increase. Data from retrospective registries demonstrated lower procedural success and higher residual PVL after TAVI in patients with bicuspid compared to tricuspid aortic valve disease (27-30). Implantation of new-generation devices yielded improved outcomes, giving rise to hope that TAVI may become a valid treatment option in bicuspid aortic valve disease in the future (30). Due to the paucity of data, guidelines favor SAVR in these patients at current (13).

\section{Morbidity and Mortality}

After early reports of increased stroke rates after TAVI (6), more recent trials have consistently demonstrated similar outcomes for mortality and stroke after TAVI or SAVR. However, distinct complication patterns have repeatedly been reported for both treatment options (see Table 1). These need to be weighed against the individual patient's risk profile when choosing the optimal treatment modality. These include a higher incidences of acute kidney injury, bleeding events, and atrial fibrillation after SAVR. TAVI was associated with faster recovery and shorter index hospitalization but a higher rate of re-interventions or heart failure were documented during follow-up. Long-term results will be essential to gain further insights into these important first observations. While major vascular complications were common after transfemoral TAVI with first-generation devices (31), a significant decrease was observed in recently reported intermediate-risk trials (810). A shift in patients' risk and device refinements with smaller delivery systems and improved vascular closure devices may be responsible for this decline. Permanent pacemaker implantation remains a concern after TAVI, particularly with self-expanding THV. Although data remain ambiguous regarding the association of pacemaker implantation and outcome after TAVI at current $(32,33)$, this issue requires in-depth evaluation, particularly in the treatment of younger patients.

Although a major advantage of TAVI relates to the less invasive procedure compared to SAVR, the risk for rare but life-threatening complications after TAVI (e.g., annular rupture, valve migration, or coronary obstruction) requiring bail-out emergency cardiac surgery must be taken into account. Recently 
published data from the European Registry on Emergent Cardiac Surgery during TAVI (EuRECS-TAVI) reported an incidence of emergent cardiac surgery of $0.7 \%$ in recent years. Most common causes were left ventricular guidewire perforations (28.3\%) and annular ruptures (21.2\%). Most of these complications occurred during the procedure and mortality remained high despite emergent cardiac surgery (34). While these serious procedure-related complications were more frequent in the early TAVI era and have become very rare events at this stage (35), expansion of TAVI towards younger and low-risk patients requires an even more critical appraisal and all measures need to be taken to prevent these complications.

\section{Cost-Effectiveness}

With the rapid growth of TAVI volume, its implications on healthcare systems and its cost-effectiveness will become even more important, particularly while expanding TAVI indications to lower risk patients (36). An early analysis from the Netherlands demonstrated higher 1-years costs of TAVI vs. SAVR in intermediate-risk patients (37). This cost difference was mainly driven by the difference in device prices. A recent cost-effectiveness analysis from the Partner 2A and Sapien 3 trials reported lower costs at 2 years after TAVI (3). Higher procedural costs were compensated for by shorter hospitalization and substantially lower costs during follow-up. Regional and national differences in reimbursement and device costs impede

\section{REFERENCES}

1. Lindroos M, Kupari M, Heikkilä J, Tilvis R. Prevalence of aortic valve abnormalities in the elderly: an echocardiographic study of a random population sample. J Am Coll Cardiol. (1993) 21:1220-5. doi: 10.1016/0735-1097(93)90249-Z

2. Thyregod HGH. Five-year outcomes from the all-comers Nordic Aortic Valve Intervention randomized clinical trial in patients with severe aortic valve stenosis. In: ACC 2018 - 67th Annual Scientific Sessions. Orlando, FL (2018).

3. Cohen DJ. Cost-effectiveness of transcatheter vs. surgical aortic valve replacement in intermediate risk patients. In: Transcatheter Cardiovascular Therapeutic. Denver, CO (2017).

4. Cribier A, Eltchaninoff H, Bash A, Borenstein N, Tron C, Bauer F, et al. Percutaneous transcatheter implantation of an aortic valve prosthesis for calcific aortic stenosis: first human case description. Circulation (2002) 106:3006-8. doi: 10.1161/01.CIR.0000047200.36165.B8

5. Beckmann A, Funkat AK, Lewandowski J, Frie M, Ernst M, Hekmat K, et al. German Heart Surgery Report 2016: the annual updated registry of the german society for thoracic and cardiovascular surgery. Thorac Cardiovasc Surg. (2017) 65:505-18. doi: 10.1055/s-0037-1606603

6. Smith CR, Leon MB, Mack MJ, Miller DC, Moses JW, Svensson LG, et al. Transcatheter versus Surgical Aortic-Valve Replacement in High-Risk Patients. N Engl J Med. (2011) 364:2187-98. doi: 10.1056/NEJMoa1103510

7. Adams DH, Popma JJ, Reardon MJ, Yakubov SJ, Coselli JS, Deeb GM, et al. Transcatheter aortic-valve replacement with a self-expanding prosthesis. $N$ Engl J Med. (2014) 370:1790-8. doi: 10.1056/NEJMoa1400590

8. Reardon MJ, Van Mieghem NM, Popma JJ, Kleiman NS, Søndergaard L, Mumtaz M, et al. Surgical or transcatheter aortic-valve replacement in intermediate-risk patients. $N$ Engl J Med. (2017) 376:1321-31. doi: 10.1056/NEJMoa1700456

9. Thyregod HG, Steinbrüchel DA, Ihlemann N, Nissen H, Kjeldsen BJ, Petursson P, et al. Transcatheter versus surgical aortic valve replacement in generalization of these results. However, health economic analyses will gain importance as the field expands.

\section{CONCLUSION}

TAVI has become the standard treatment in patients at increased surgical risk and is increasingly being performed in patients at intermediate to low risk at current. Non-inferiority has been demonstrated in different intermediate risk cohorts. However, before broad expansion to lower risk and younger patients can be recommended, several challenges-particularly with regard to valve durability - need to be addressed. Several randomized trials are under way to investigate these issues and will determine future guideline recommendations. For now, distinct risks should be weighed into the decision of TAVI vs. SAVR, incorporating each patient's individual risk profile and personal preferences. Shared-decision making will increasingly become a crucial element in this process. Preferences of the informed patient should be discussed, balanced, and weighed into the joint treatment decision of the interdisciplinary heart team to select the appropriate treatment for every individual patient while expanding TAVI to intermediate and low risk operative patients.

\section{AUTHOR CONTRIBUTIONS}

LV: literature research, drafted the manuscript; MS: literature research, critical revision of the manuscript. patients with severe aortic valve stenosis: one-year results from the all-comers Nordic Aortic Valve Intervention (NOTION) randomized clinical trial. J Am Coll Cardiol. (2015) 65:2184-94. doi: 10.1016/j.jacc.2015.03.014

10. Leon MB, Smith CR, Mack MJ, Makkar RR, Svensson LG, Kodali SK, et al. Transcatheter or surgical aortic-valve replacement in intermediate-risk patients. N Engl J Med. (2016) 374:1609-20. doi: 10.1056/NEJMoa1514616

11. Dewey TM, Brown D, Ryan WH, Herbert MA, Prince SL, Mack MJ. Reliability of risk algorithms in predicting early and late operative outcomes in highrisk patients undergoing aortic valve replacement. J Thorac Cardiovasc Surg. (2008) 135:180-7. doi: 10.1016/j.jtcvs.2007.09.011

12. Silaschi M, Conradi L, Seiffert M, Schnabel R, Schön G, Blankenberg S, et al. Predicting risk in transcatheter aortic valve implantation: comparative analysis of EuroSCORE II and established risk stratification tools. Thorac Cardiovasc Surg. (2014) 63:472-8. doi: 10.1055/s-0034-1389107

13. Baumgartner H, Falk V, Bax JJ, De Bonis M, Hamm C, Holm PJ, et al. 2017 ESC/EACTS Guidelines for the management of valvular heart disease. Eur Heart J. (2017) 38:2739-91. doi: 10.1093/eurheartj/ehx391

14. Nishimura RA, Otto CM, Bonow RO, Carabello BA, Erwin JP III, Fleisher LA, et al. 2017 AHA/ACC focused update of the 2014 AHA/ACC guideline for the management of patients with valvular heart disease. J Am Coll Cardiol. (2017) 70:252-89. doi: 10.1016/j.jacc.2017.03.011

15. Eggebrecht H, Mehta RH. Transcatheter aortic valve implantation (TAVI) in Germany 2008-2014: on its way to standard therapy for aortic valve stenosis in the elderly? Eurointervention (2016) 11:1029-33. doi: 10.4244/EIJY15 M09_11

16. Walther T, Hamm CW, Schuler G, Berkowitsch A, Kötting J, Mangner $\mathrm{N}$, et al. Perioperative results and complications in 15,964 transcatheter aortic valve replacements. J Am Coll Cardiol. (2015) 65:2173-80. doi: 10.1016/j.jacc.2015.03.034

17. Grover FL, Vemulapalli S, Carroll JD, Edwards FH, Mack MJ, Thourani VH, et al. 2016 Annual Report of the Society of Thoracic Surgeons/American College of Cardiology Transcatheter Valve Therapy 
Registry. J Am Coll Cardiol. (2017) 69:1215-30. doi: 10.1016/j.jacc.2016.1 1.033

18. Nielsen HH, Klaaborg KE, Nissen H, Terp K, Mortensen PE, Kjeldsen BJ, et al. A prospective, randomised trial of transapical transcatheter aortic valve implantation vs. surgical aortic valve replacement in operable elderly patients with aortic stenosis: the STACCATO trial. Eurointervention (2012) 8:383-9. doi: 10.4244/EIJV8I3A58

19. Douglas PS, Leon MB, Mack MJ, Svensson LG, Webb JG, Hahn RT, et al. Longitudinal hemodynamics of transcatheter and surgical aortic valves in the PARTNER trial. JAMA Cardiol. (2017) 2:1197-206. doi: 10.1001/jamacardio.2017.3306

20. Arora S, Ramm CJ, Strassle PD, Vaidya SR, Caranasos TG, Vavalle JP, et al. Review of major registries and clinical trials of late outcomes after transcatheter aortic valve replacement. Am J Cardiol. (2017) 120:331-6. doi: 10.1016/j.amjcard.2017.04.029

21. Thourani VH, Kodali S, Makkar RR, Herrmann HC, Williams M, Babaliaros $\mathrm{V}$, et al. Transcatheter aortic valve replacement versus surgical valve replacement in intermediate-risk patients: a propensity score analysis. Lancet (2016) 387:2218-25. doi: 10.1016/S0140-6736(16)30073-3

22. Chakravarty T, Søndergaard L, Friedman J, De Backer O, Berman D, Kofoed KF, et al. Subclinical leaflet thrombosis in surgical and transcatheter bioprosthetic aortic valves: an observational study. Lancet (2017) 389:238392. doi: $10.1016 / \mathrm{S} 0140-6736(17) 30757-2$

23. Dvir D, Bourguignon T, Otto CM, Hahn RT, Rosenhek R, Webb JG, et al. Standardized definition of structural valve degeneration for surgical and transcatheter bioprosthetic aortic valves. Circulation (2018)137:388-99. doi: 10.1161/CIRCULATIONAHA.117.030729

24. Capodanno D, Petronio AS, Prendergast B, Eltchaninoff H, Vahanian A, Modine T, et al. Standardized definitions of structural deterioration and valve failure in assessing long-term durability of transcatheter and surgical aortic bioprosthetic valves: a consensus statement from the European Association of Percutaneous Cardiovascular Interven. Eur Heart J. (2017) 38:3382-90. doi: $10.1093 /$ ejcts/ezx244

25. Johnston DR, Soltesz EG, Vakil N, Rajeswaran J, Roselli EE, Sabik JF III, et al. Long-term durability of bioprosthetic aortic valves: implications from 12,569 implants. Ann Thorac Surg. (2015) 99:1239-47. doi: 10.1016/j.athoracsur.2014.10.070

26. Dvir D, Webb JG, Bleiziffer S, Pasic M, Waksman R, Kodali S, et al. Transcatheter aortic valve implantation in failed bioprosthetic surgical valves. JAMA (2014) 312:162-70. doi: 10.1001/jama.2014.7246

27. Bauer $T$, Linke A, Sievert H, Kahlert P, Hambrecht R, Nickenig G, et al. Comparison of the effectiveness of transcatheter aortic valve implantation in patients with stenotic bicuspid versus tricuspid aortic valves (from the German TAVI Registry). Am J Cardiol. (2014) 113:518-21. doi: 10.1016/j.amjcard.2013.10.023

28. Abdel-Wahab M, Zahn R, Horack M, Gerckens U, Schuler G, Sievert H, et al. Aortic regurgitation after transcatheter aortic valve implantation: incidence and early outcome. Results from the German transcatheter aortic valve interventions registry. Heart (2011) 97:899-906 doi: 10.1136/hrt.2010.217158

29. Mylotte D, Lefevre T, Søndergaard L, Watanabe Y, Modine T, Dvir D, et al. Transcatheter aortic valve replacement in bicuspid aortic valve disease. J Am Coll Cardiol. (2014) 64:2330-9. doi: 10.1016/j.jacc.2014. 09.039

30. Yoon SH, Bleiziffer S, De Backer O, Delgado V, Arai T, Ziegelmueller J, et al. Outcomes in transcatheter aortic valve replacement for bicuspid versus tricuspid aortic valve stenosis. J Am Coll Cardiol. 69:2579-89 (2017). doi: 10.1016/j.jacc.2017.03.017

31. Généreux P, Webb JG, Svensson LG, Kodali SK, Satler LF, Fearon WF, et al. Vascular complications after transcatheter aortic valve replacement. J Am Coll Cardiol. (2012) 60:1043-52. doi: 10.1016/j.jacc.2012.07.003

32. Nazif TM, Dizon JM, Hahn RT, Xu K, Babaliaros V, Douglas PS, et al. Predictors and clinical outcomes of permanent pacemaker implantation after transcatheter aortic valve replacement. JACC Cardiovasc Interv. (2015) 8:60-9. doi: $10.1016 /$ j.jcin.2014.07.022

33. Buellesfeld L, Stortecky S, Heg D, Hausen S, Mueller R, Wenaweser P, et al. Impact of permanent pacemaker implantation on clinical outcome among patients undergoing transcatheter aortic valve implantation. J Am Coll Cardiol. (2012) 60:493-501. doi: 10.1016/j.jacc.2012. 03.054

34. Eggebrecht H, Vaquerizo B, Moris C, Bossone E, Lämmer J, Czerny M, et al. Incidence and outcomes of emergent cardiac surgery during transfemoral transcatheter aortic valve implantation (TAVI): insights from the European Registry on Emergent Cardiac Surgery during TAVI (EuRECS-TAVI). Eur Heart J. (2018) 39:676-84. doi: 10.1093/eurheartj/ehx713

35. Wendler O, Schymik G, Treede H, Baumgartner H, Dumonteil N, Ihlberg L, et al. SOURCE 3 registry: design and 30-day results of the European postapproval registry of the latest generation of the SAPIEN 3 Transcatheter heart valve. Circulation (2017) 135:1123-32. doi: 10.1161/CIRCULATIONAHA.116.025103

36. Reynolds MR, Baron SJ, Cohen DJ. Economic implications of transcatheter aortic valve replacement in patients at intermediate surgical risk. Circulation (2016) 134:1416-8. doi: 10.1161/CIRCULATIONAHA.116.0 21962

37. Osnabrugge RL, Head SJ, Genders TS, Van Mieghem NM, De Jaegere PP, van der Boon RM, et al. Costs of transcatheter versus surgical aortic valve replacement in intermediate-risk patients. Ann Thorac Surg. (2012) 94:195460. doi: 10.1016/j.athoracsur.2012.07.002

Conflict of Interest Statement: MS received travel compensation from Edwards Lifesciences and lecture fees from Medtronic, serves as coordinating investigator for the DEDICATE-DZHK6 trial.

The remaining author declares that the research was conducted in the absence of any commercial or financial relationships that could be construed as a potential conflict of interest.

Copyright (๑) 2018 Voigtländer and Seiffert. This is an open-access article distributed under the terms of the Creative Commons Attribution License (CC BY). The use, distribution or reproduction in other forums is permitted, provided the original author(s) and the copyright owner(s) are credited and that the original publication in this journal is cited, in accordance with accepted academic practice. No use, distribution or reproduction is permitted which does not comply with these terms. 